Detecting anharmonicity at a glance

This content has been downloaded from IOPscience. Please scroll down to see the full text. 2014 Eur. J. Phys. 35065012

(http://iopscience.iop.org/0143-0807/35/6/065012)

View the table of contents for this issue, or go to the journal homepage for more

Download details:

IP Address: 159.149.193.152

This content was downloaded on 23/07/2015 at 07:52

Please note that terms and conditions apply. 


\title{
Detecting anharmonicity at a glance
}

\author{
M Giliberti ${ }^{1}$, M Stellato ${ }^{1}$, S Barbieri ${ }^{1}$, M Cavinato $^{1}$, \\ E Rigon $^{2}$ and $M$ Tamborini ${ }^{3}$
}

\author{
${ }^{1}$ Dipartimento di Fisica, Università degli Studi di Milano, Italy \\ ${ }^{2}$ Liceo Scientifico 'G B Grassi' Saronno, Italy \\ ${ }^{3}$ Liceo Scientifico 'P Bottoni' Milano, Italy \\ E-mail: marco.giliberti@unimi.it
}

Received 23 June 2014, revised 5 August 2014

Accepted for publication 7 August 2014

Published 9 September 2014

\begin{abstract}
Harmonic motion is generally presented in such a way that most of the students believe that the small oscillations of a body are all harmonic. Since the situation is not actually so simple, and since the comprehension of harmonic motion is essential in many physical contexts, we present here some suggestions, addressed to undergraduate students and pre-service teachers, that allow one to find out at a glance the anharmonicity of a motion. Starting from a didactically motivated definition of harmonic motion, and stressing the importance of the interplay between mathematics and experiments, we give a four-point criterion for anharmonicity together with some emblematic examples. The role of linear damping is also analysed in relation to the gradual changing of harmonicity into anharmonicity when the ratio between the damping coefficient and the zero-friction angular frequency increases.
\end{abstract}

Keywords: oscillations, anharmonic motion, data logging, video analysis

\section{Introduction}

Our lab experience with graduate students in mathematics shows that the comprehension of the link between mathematics and physics in the study of oscillations is far from clear. This fact prevents them grasping the importance of the harmonic motion as a conceptual organizer that should emerge from the choice/recognition of particular deep similarities/diversities among different types of periodic motions. The kinematic definition of harmonic motion is not enough to understand the physics implied; the dynamic definition, which stems from the analysis of the potential energy, is often ineffective.

In the following, we propose an approach to harmonic motion that allows one to realize at a glance the anharmonicity/harmonicity of an oscillation and to understand the link with the mathematical aspects of the problem. The goal of this paper is to make students able to 


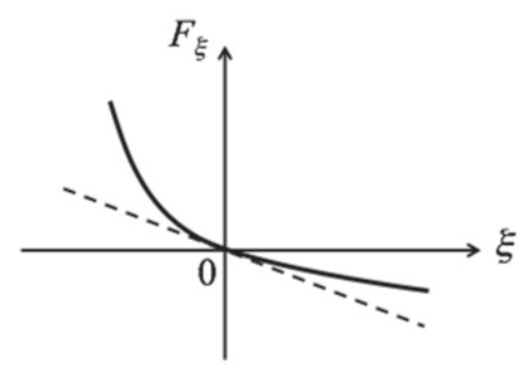

Figure 1. A restoring force and its linearization in the origin.

recognize if a motion is harmonic or not even without knowing the exact expression of the acting forces, but simply by watching the oscillations and sometimes by listening to the sound generated by the oscillations themselves. We also discuss the role of both the constant and of the linear damping in relation to the concepts of anharmonicity/harmonicity.

\section{Definition of harmonic motion}

Let us consider a one degree of freedom system subject to a restoring force, that is, a force that gives rise to a motion with a stable equilibrium point. Let us call $\xi$ the curvilinear coordinate measuring the oriented distance along the trajectory described by the moving body, with the zero corresponding to the equilibrium position. In this case, at least in a neighbourhood of $\xi=0$, the graph of the $\xi$-component of the restoring force, $F_{\xi}, v s \xi$ lies in the second and in the fourth quadrant (solid line in figure 1). Moreover, if the restoring force is sufficiently regular, that is, the function $F_{\xi}(\xi)$ is continuous and differentiable in the origin, it can be approximated by its tangent line in that point, provided the amplitude of the oscillation is small enough (dashed line in figure 1).

As a consequence, a body subject to a sufficiently regular restoring force with the first derivative different from zero in the equilibrium point, for small amplitude oscillations, will obey the equation of motion:

$$
F_{\xi}=-k \xi, \quad(k>0) .
$$

Denoting with $m$ the mass of the body, we immediately get

$$
\ddot{\xi}+\omega_{0}^{2} \xi=0
$$

where

$$
\omega_{0} \equiv \sqrt{\frac{k}{m}} .
$$

We define harmonic motion as the motion of a point mass satisfying equation (1) or, equivalently, equation (2). This is an intrinsic definition, in the sense that, once a reference frame is fixed, it refers only to the trajectory and to the resultant acting force, which are intrinsic characteristics of the motion. In many cases, however, it is useful to perform an invertible regular transformation,

$$
\xi=\xi(q)
$$

from the coordinate $\xi$ to a new coordinate $q$ (in general, an angle or a position on a linear axis) that is more suitable to discuss lab experiments or to make a mathematical model of the 
system. Since equation (2) is a linear equation, if we require that the transformation (4) preserves the description of the motion, or equivalently preserves the form of equation (2), the only allowed tranformations will be the linear ones. Nonetheless, since in most situations we are mainly interested in the small oscillations around the equilibrium point, there is no need for the whole tranformation (4) to be linear. It is enough that it can be linearized in the origin; namely, that in the neighborhood of $q=0, \xi(q)$ it can be approximated to the first order in $q$ by:

$$
\xi(q)=\frac{\mathrm{d} \xi}{\mathrm{d} q}(0) \cdot q ; \quad \frac{\mathrm{d} \xi}{\mathrm{d} q}(0) \neq 0 .
$$

Equation (2) can be easily integrated to obtain the general solution [1-3]:

$$
\xi(t)=A \cos \left(\omega_{0} t+\varphi_{0}\right),
$$

where the amplitude $A$ and the initial phase $\varphi_{0}$ are two integration constants that are completely independent of the value of the (angular) frequency $\omega_{0}$, which is solely determined by dynamic conditions. The stated independence of $A$ from $\omega_{0}$ is generally referred to as isochronism.

We would like to stress that the harmonic motion defined earlier is not necessarily rectilinear, as $\xi$ is a curvilinear coordinate (for instance, the ends of a torsional pendulum describe an arc of circumference performing harmonic oscillations over a wide range of angles). It is also important to emphasize that $F_{\xi}$ must not be confused with the intensity of the total acting force, but it is only its component along the direction of motion. This is a conceptual aspect for which particular care is needed in describing motions on curved trajectories. In fact, in these cases, the resultant force is different from zero even in the equilibrium position because the contribution of the centripetal component has to be considered; while, on the contrary, $F_{\xi}$ is indeed null.

In conclusion, the path toward the previous definition leads us to formulate a four-point criterion saying that the small oscillations of a one degree of freedom system are harmonic if $\xi=0$

(a) is a stable equilibrium point; and in $\xi=0$ :

(b) the function $F_{\xi}(\xi)$ is continuous;

(c) the function $F_{\xi}(\xi)$ is differentiable;

(d) $\frac{\mathrm{d} F_{\xi}}{\mathrm{d} \xi} \neq 0$.

Obviously, condition (c) implies condition (b). Nevertheless, we believe that, from a didactical point of view, keeping these conditions separate allows a clearer comprehension of the physics involved.

\section{Harmonic or not?}

The prototype of harmonic motion is the mass-spring oscillator that has been discussed in many papers and textbooks [1-6]. An analysis of the motion of a one degree of freedom vertical mass-spring oscillator can be done via sonar detection and the 'Logger Pro' software [7]. In figure 2, the acceleration $v s$ position is shown. It will not be discussed here, but it will be used in the following as the gold standard in analyzing other oscillations.

Let us now discuss how the mathematical conditions (a) to (d) of our four-point criterion are linked to real experiments and how the anharmonicity/harmonicity of small amplitude oscillations can be in many cases decided 'on sight,' even without explicitly knowing the 


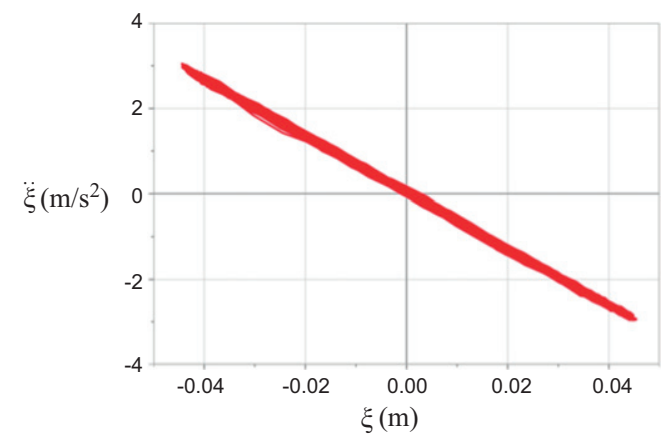

Figure 2. Vertical mass-spring oscillator. Measured acceleration vs position: the graph represents a typical restoring force. The data have been collected and processed by a 'Logger Pro' sonar system.

(a)

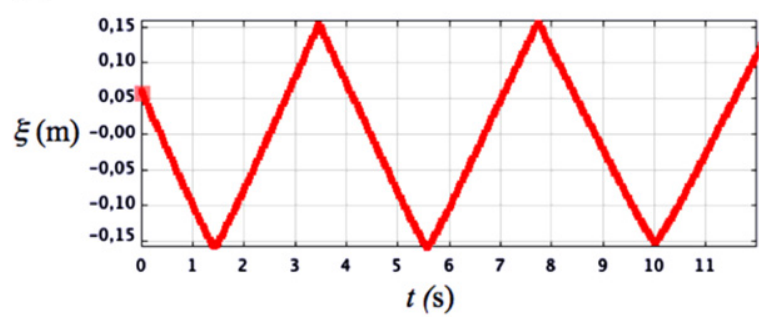

(b)

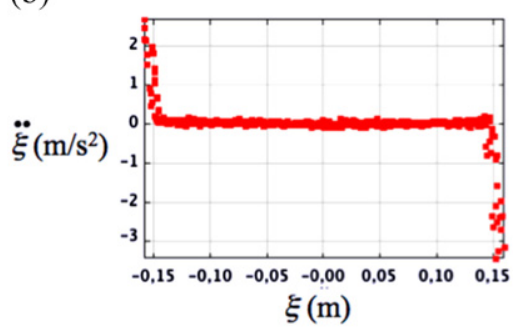

Figure 3. Bouncing disk. (a) Measured position vs time; (b) Measured acceleration vs position. The data have been processed by the 'Tracker' video analysis software.

equation of motion. Listening to the sound produced by the oscillations can sometimes help us discriminate between anharmonicity and harmonicity. Let us consider some examples.

\subsection{Bouncing disk}

The bouncing disk consists of a disk moving back and forth between two elastic edges of an air table. Are these oscillations harmonic? No, they are not, because the system has no single stable equilibrium point, but an infinite set of neutral equilibrium positions; a fact that implies that we cannot even introduce the notion of small oscillations. In this case, the first point of our criterion is not satisfied. A video of the motion has been analyzed by the software 'Tracker' [8]. The results are shown in figure 3.

While in the case of the mass-spring oscillator the acceleration $v s$ position diagram is a straight line lying in the second and fourth quadrant passing through the origin of the axes, in the case of the bouncing disk, such a diagram is completely flat, except at the edges where the force is impulsive (figure 3(b)). In fact, the position $v$ s time graph shows that we are in the presence of a uniform rectilinear motion between the turning points (figure 3(a)).

\subsection{Galileo oscillator}

Let us consider a V-shaped track, as in figure 4, with a ball rolling over it, a device that is sometimes referred to as the Galileo oscillator [9]. The function $F_{\xi}(\xi)$ is not continuous in 


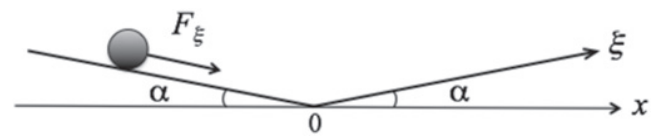

Figure 4. The Galileo oscillator. The $\xi$-coordinate along the trajectory and the $x$-axis along which the motion is detected.
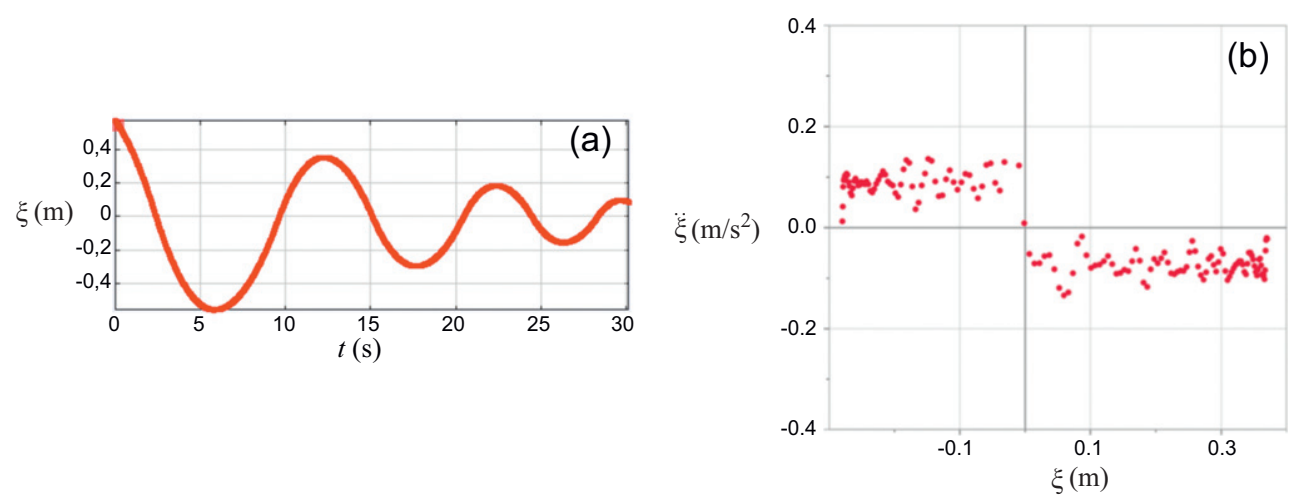

Figure 5. The Galileo oscillator. (a) Position vs time; (b) Acceleration vs position. The data have been processed by the 'Tracker' video analysis software; they show two different values of the acceleration, as can be inferred from equation (7).

$\xi=0$; therefore, the second point of our criterion is not fulfilled and the motion cannot be harmonic.

In fact, neglecting both the friction and the rolling energy, which indeed are always present in a real experiment, the force acting on an object of mass $m$ along this kind of trajectory is given by

$$
m g \sin \alpha ; \xi<0 \quad-m g \sin \alpha ; \xi>0 .
$$

If the angle $\alpha$ is sufficiently small so that the ball can really oscillate back and forth between the two parts of the track, we can make a video analysis of the motion. The results are shown in figure 5. The coordinate $q=x$ is used instead of $\xi$; the transformation (4) can be written as

$$
\xi(x)=\frac{x}{\cos \alpha},
$$

that is clearly a linear transformation and, as already said, preserves the harmonicity/ anharmonicity of the motion.

The motion is much more similar to that of a freely falling bouncing ball than to that of a mass-spring oscillator, and it cannot be approximated by a harmonic motion, however small the amplitude of oscillation.

\subsection{The interrupted pendulum}

Let us analyse a simple pendulum of length $l_{1}$ that is interrupted in its motion by a peg between the point of suspension and the equilibrium point, so that its length abruptly changes to $l_{2}$ (see figure 6(a)) [10]. The equation of motion is 

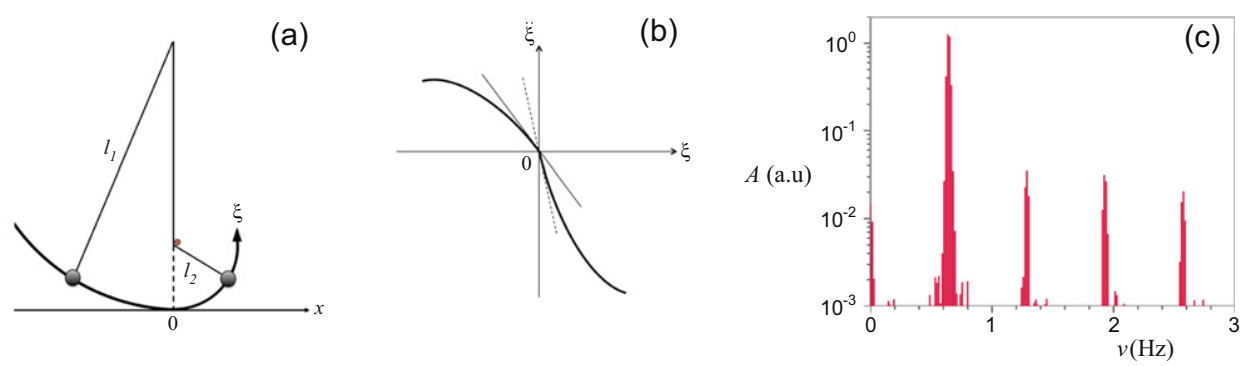

Figure 6. The interrupted pendulum. (a) The apparatus: the bob is shown at two different times; (b) acceleration $v$ s position; (c) FFT of the motion waveform. The data for the FFT have been collected and processed by a 'Logger Pro' system.

$$
\ddot{\xi}+g \sin \frac{\xi}{l_{1}}=0 ; \quad \xi \leqslant 0 \quad \ddot{\xi}+g \sin \frac{\xi}{l_{2}}=0 ; \quad \xi>0,
$$

which shows that $\xi=0$ is a corner point of the function $\ddot{\xi}(\xi)$ (figure $6(\mathrm{~b})$ ). Therefore, the function $F_{\xi}(\xi)$ is not differentiable in $\xi=0$, the third point of our criterion does not hold, and the motion of the pendulum cannot be approximated by a harmonic motion, not even in the small oscillation limit.

For what concerns the period $T_{12}$ of the small oscillations of this asymmetric pendulum, we obviously have

$$
T_{12}=\frac{T_{1}}{2}+\frac{T_{2}}{2}
$$

where $T_{1}$ and $T_{2}$ are the oscillation periods of the two pendulums of length $l_{1}$ and $l_{2}$, respectively. Consequently, the interrupted pendulum is isochronous for small angles of oscillation without being harmonic. The fast Fourier transform (FFT) of the motion waveform (figure 6(c)) shows clearly, even for small oscillations, the first harmonic of frequency $\nu_{1}=1 / T_{12}$ and some of its multiples, as one expects for a non-sinusoidal periodic motion of period $T_{12}$, at variance to what one could naively expect, i.e., the presence of the two frequencies $1 / T_{1}$ and $1 / T_{2}$. We stress that the request of differentiability of the function $F_{\xi}(\xi)$ in the origin, which at first might seem a mere mathematical question, corresponds to detectable physical effects.

We want also to observe that another case in which the function $F_{\xi}(\xi)$ is not differentiable in $\xi=0$ is when it has a vertical tangent line in that point. Physically, we can think of a very tough spring that we are not able to stretch with our lab equipment, so that the motion of one of its ends cannot even occur.

\subsection{The $x^{4}$-track}

Here we want to analyse the case when only the condition (d) of our four-point criterion is not satisfied, that is, $\frac{\mathrm{d}}{\mathrm{d} \xi} F_{\xi}(0)$ is zero. If the force and its first derivative are zero in the origin, the second derivative $\frac{\mathrm{d}^{2}}{\mathrm{~d} \xi^{2}} F_{\xi}(0)$ must also be zero; otherwise, $\xi=0$ would not be a stable equilibrium point. Thus, the first term different from zero in the MacLaurin expansion of $F_{\xi}$ must be at least proportional to $\xi^{3}$. We want to show that, in this condition, the motion is not only anharmonic, but also not isochronous.

Let $U(\xi)$ be the potential energy of the ball of mass $m$. Energy conservation can be written as 


$$
\frac{1}{2} m \dot{\xi}^{2}+U(\xi)=E
$$

where $E$ is the total constant energy of the system. Let $A_{1}$ and $A_{2}$ be the extremes of oscillation (solution of $U(\xi)=E$ ); then the period of oscillation $T$ is twice the time spent by the body for going from $A_{1}$ to $A_{2}$. That is

$$
T=2 \int_{A_{1}}^{A_{2}} \frac{\mathrm{d} \xi}{\sqrt{\frac{2}{m}[E-U(\xi)]}} .
$$

In our case, $U(\xi)=c \xi^{4}$, where $c$ is a positive constant. Therefore, putting $A_{1} \equiv-A$ and $A_{2} \equiv A$, with $c A^{4}=E$, we obtain

$$
T=2 \sqrt{\frac{m}{2}} \int_{-A}^{A} \frac{\mathrm{d} \xi}{\sqrt{c A^{4}-c \xi^{4}}}=4 \sqrt{\frac{m}{2 c}} \int_{0}^{A} \frac{\mathrm{d} \xi}{\sqrt{A^{4}-\xi^{4}}} .
$$

Making the substitution $\xi \equiv A x, T$ is given by

$$
T=4 \sqrt{\frac{m}{2 c}} \frac{1}{A} \int_{0}^{1} \frac{\mathrm{d} x}{\sqrt{1-x^{4}}}=4 \sqrt{\frac{m}{2 c}} \alpha \frac{1}{A},
$$

where the constant

$$
\alpha \equiv \int_{0}^{1} \frac{\mathrm{d} x}{\sqrt{1-x^{4}}} \sim 1.31
$$

has been numerically calculated. The period of oscillation depends on the amplitude as $A^{-1}$ and, therefore, it is not isochronous however small the amplitude $A$. From equation (14), we observe that when $A \rightarrow 0, T \rightarrow \infty$. To better understand this behavior, we note that the linearized equation (2) reads

$$
\ddot{\xi}=0 \text {, }
$$

a fact that makes a small neighborhood of $\xi=0$ similar to a region of neutral equilibrium.

In order to have a mechanical example of this kind of motion, one can consider a ball moving on a $x^{4}$-shaped track. One observes that about the equilibrium point the track is nearly flat and realizes that, in order to have only small amplitude oscillations, the ball has to be substantially at rest with 'long' oscillation periods. On the contrary, to get periods of oscillation that are not very long, one is forced to consider sufficiently great amplitudes. In this case, by just listening to the sound produced by the sliding ball, one can infer that the motion is manifestly anharmonic.

Similar considerations can be made more generally when the first term in the MacLaurin expansion of $F_{\xi}(\xi)$ is proportional to a generic $\xi^{2 n-1}$ with $n>1$; the dependence of the period $T$ on the amplitude $A$ would be $T \propto A^{-(n-1)}$.

\section{Damped oscillations}

In addition to the conditions previously discussed, a useful way to recognize anharmonicity is to find an amplitude dependence of the period of oscillation. Since real motions are always damped, we have to exclude that this dependence comes from damping instead of being due to an intrinsic anharmonicity.

In the interesting case of sliding friction, it can be demonstrated that a constant friction force does not affect the frequency of a simple harmonic motion, but only the amplitude of 
oscillation, which is decreased each cycle $[11,12]$. Therefore sliding friction cannot produce an amplitude dependence of the oscillation period.

In the case of viscous friction, the restoring force no longer depends only on position, but also on velocity and the situation is a little bit more complicated. In the simplest case, for small velocities, the damping can be assumed to be linear with the velocity, and the equation of motion is therefore

$$
\ddot{\xi}+\omega_{0}^{2} \xi+2 \gamma \dot{\xi}=0
$$

where $\gamma$ is the damping coefficient and $\omega_{0}$ is the zero-friction angular frequency. In the case of neither critical $\left(\gamma=\omega_{0}\right)$ nor over-damped $\left(\gamma>\omega_{0}\right)$ motion (which we do not consider here), the solution of equation (17) is

$$
\xi(t)=A \mathrm{e}^{-\gamma t} \cos \left(\omega t+\varphi_{0}\right) ; \quad \omega \equiv \omega_{0} \sqrt{1-\left(\frac{\gamma}{\omega_{0}}\right)^{2}} ; \quad \gamma<\omega_{0},
$$

where $A$ and $\varphi_{0}$ are integration constants and $\omega$ is the actual angular frequency of the damped harmonic motion. To be more precise, since the motion is not, strictly speaking, periodic, instead of the period we should refer to the notion of pseudo-frequency (and of pseudoperiod).

It is important to deeply understand the physical meaning of the constants appearing in equation (18). Let us consider the problem of writing down the explicit solution of equation (17) for an oscillator that starts from rest at the initial position $\xi(0)=\xi_{0}$. For that, we have to solve the Cauchy problem:

$$
\ddot{\xi}+\omega_{0}^{2} \xi+2 \gamma \dot{\xi}=0 ; \quad \xi(0)=\xi_{0}, \quad \dot{\xi}(0)=0 .
$$

With simple calculations, we obtain the constants $A$ and $\varphi_{0}$,

$$
\frac{A}{\xi_{0}}=\left[\frac{1}{1-\left(\frac{\gamma}{\omega_{0}}\right)^{2}}\right]^{\frac{1}{2}} ; \quad \varphi_{0}=-\arctan \frac{\frac{\gamma}{\omega_{0}}}{\left[1-\left(\frac{\gamma}{\omega_{0}}\right)^{2}\right]^{\frac{1}{2}}}
$$

where the relation on the left shows that $A$ is always greater than $\xi_{0}$. We can observe that, although $A$ depends on $\xi_{0}$ and on the ratio $\gamma / \omega_{0}$, the pseudo-frequency does not depend on $\xi_{0}$ and, therefore, a pseudo-period dependence on the amplitude cannot come from linear damping. Furthermore, the fact that $\varphi_{0}$ is negative implies that the elapsed time $T_{Q}$ between the start $(t=0)$ and the first passage through the equilibrium position is more than a quarter of a period, namely,

$$
T_{Q}=\frac{T}{4}-\frac{T \varphi_{0}}{2 \pi}
$$

while the time interval between the equilibrium position and the first maximum is less than $T / 4$; the period of a complete oscillation is $T=2 \pi / \omega$. From equations (18) and (20), we obtain the fractional increase

$$
I_{Q}=\frac{T_{Q}-\frac{T}{4}}{\frac{T}{4}}=\frac{2}{\pi} \arctan \frac{\gamma / \omega_{0}}{\sqrt{1-\left(\frac{\gamma}{\omega_{0}}\right)^{2}}},
$$



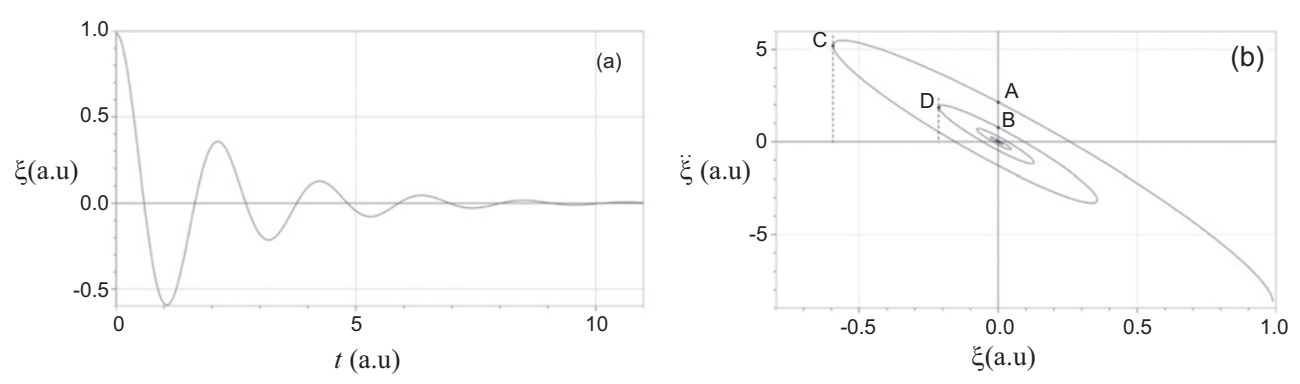

Figure 7. The damped harmonic oscillator. (a) Simulated amplitude decay with a ratio $\gamma / \omega_{0} \sim 0.16$; (b) Simulated acceleration $v s$ position with the same ratio $\gamma / \omega_{0} \sim 0.16$ as in panel (a).

that can be seen as an estimate of the 'anharmonicity degree' of the motion. The moving towards the equilibrium position and the moving away from the equilibrium position are not symmetric, because in the former case, the friction force is antiparallel to the restoring force while, in the latter, it is parallel.

From equation (18) it is straightforward to obtain

$$
\ddot{\xi}=-\left(\omega^{2}-\gamma^{2}\right) \xi+2 \gamma \omega A e^{-\gamma t} \sin \left(\omega t+\varphi_{0}\right),
$$

which shows that the acceleration is the sum of two contributions: a harmonic-like term, plus a temporal damped sinusoidal term. The first term in equation (23) could be surprising because the coefficient of $\xi$ is not $\omega^{2}$, as one could at first expect. The fact is that the pseudofrequency must not be confused with the frequency. While frequency is a property of a periodic motion that, for a harmonic oscillator, is fixed by the slope of the acceleration $v s$ position graph, the pseudo-frequency is the rhythm given by the oscillator, which performs a complete oscillation around the origin.

The analysis of the graphs of the acceleration $v s$ position allows us to understand the differences between the harmonic and the linearly damped harmonic motions.

Let us first analyse the case $\gamma T=1$, when the amplitude of oscillation is reduced by a factor $1 / e$ in a period so that some complete oscillations are still easily visible, though clearly damped. In this case, we have:

$$
\frac{\gamma}{\omega_{0}}=\left(4 \pi^{2}+1\right)^{-\frac{1}{2}} \sim 0.16,
$$

and $I_{Q} \sim 0.1$ In figure 7(a), a plot of $\xi$ vs $t$ shows four complete oscillations. Panel (b) of figure 7 displays $\ddot{\xi}$ vs $\xi$ and gives us a picture of the general structure of the acceleration $v s$ displacement for the so-called damped harmonic motions. When the body passes through the equilibrium position, the harmonic force (see first term on the right side of equation (23) is indeed zero, but the viscous drag opposes the direction of motion, so that the curve $F_{\xi}(\xi)$ (that is no more a single valued function of $\xi$ ) does not touch the origin, except when the motion stops). In figure 7(b), the pseudo-period is the time taken to go from point A to point B or, equivalently, from the extremes $\mathrm{C}$ and $\mathrm{D}$. It is this interval of time that remains constant during the oscillation.

It is interesting to analyse how the physical behavior of the damped oscillator changes for smaller or higher values of $\gamma / \omega_{0}$. 

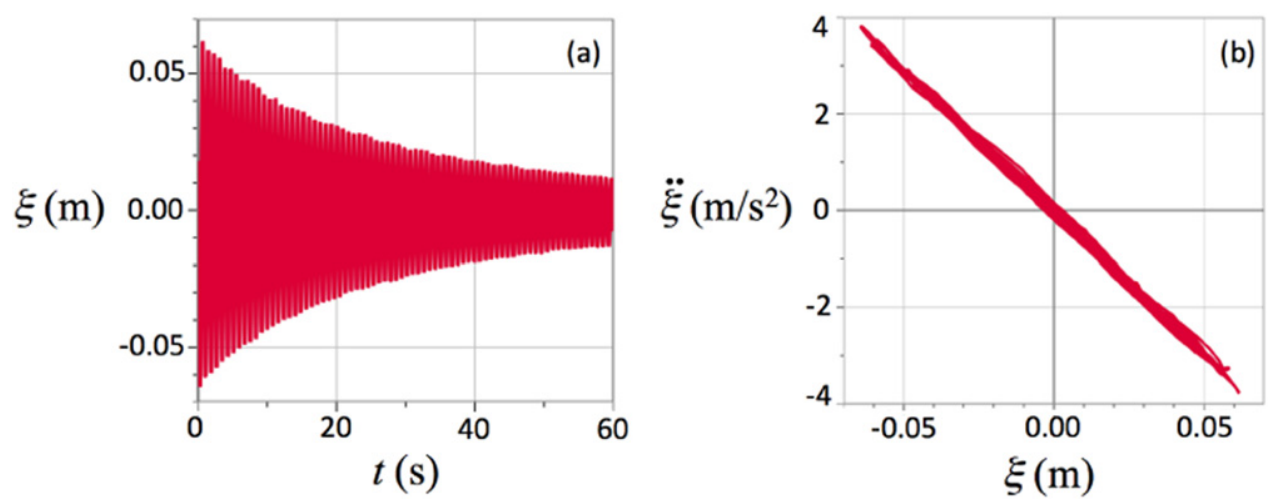

Figure 8. The damped harmonic oscillator (with a CD-rom stuck to the mass). (a) Measured amplitude decay with a ratio $\gamma / \omega_{0} \sim 0.0045$; (b) measured acceleration $v s$ position with the same ratio $\gamma / \omega_{0} \sim 0.0045$ as in panel (a). The data have been collected and processed by a 'Logger Pro' sonar system.
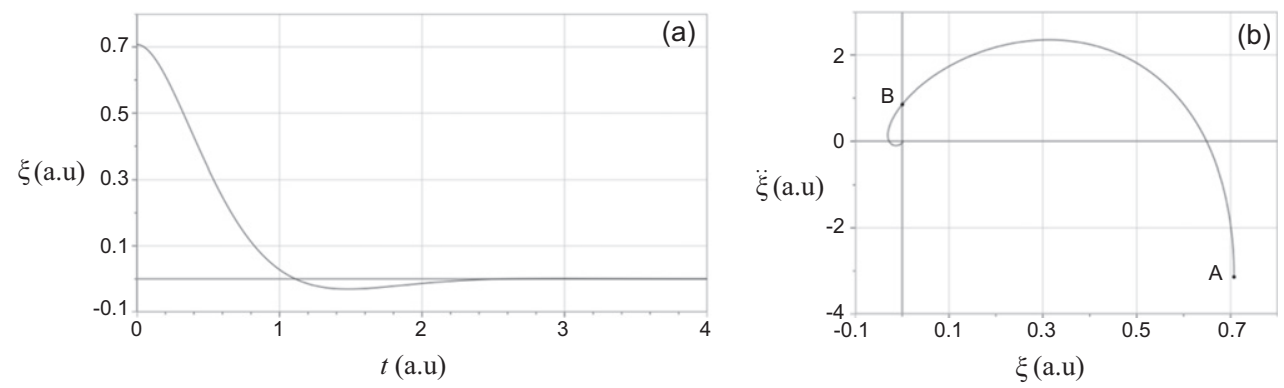

Figure 9. The damped harmonic oscillator. (a) Simulated amplitude decay with a ratio $\gamma / \omega_{0}=1 / \sqrt{2}$; (b) Simulated acceleration $v s$ position with the same ratio $\gamma / \omega_{0}=1 / \sqrt{2}$ as in panel (a).

For $\gamma / \omega_{0} \ll 1$

$$
I_{Q} \sim \frac{2}{\pi} \frac{\gamma}{\omega_{0}} \ll 1, \quad \text { and } \quad T_{Q} \sim T / 4 .
$$

Moreover,

$$
\omega \sim \omega_{0}\left[1-\frac{1}{2}\left(\frac{\gamma}{\omega_{0}}\right)^{2}\right],
$$

a quantity that is difficult to distinguish from $\omega_{0}$ in most simple lab experiments. In figure 8 , a real damped mass-spring oscillator is analysed. In this case, $\gamma / \omega_{0} \sim 0.0045$ and $I_{Q} \sim 0.003$. While the damping has a clear and visible effect on the amplitude (see figure 8(a)), since $\omega_{0} \sim 7.73 \mathrm{~s}^{-1}$, from equation (26) it follows that, in order to observe the increase of $T_{Q}$ with respect to $T / 4$, we should measure time with an accuracy of $10^{-4} \mathrm{~s}$ that exceeds most of the standard didactical lab devices. The acceleration $v s$ position plot has the same structure of that of figure 7(b), but is completely squeezed so that, to resolve the coils of the spiral, one should have highly precise tools. 
From equation (23) we can infer that for $\gamma>\omega$ the first term on the right side changes sign; therefore, the harmonic term of the force is substituted by a moving away one. If $\gamma=\omega$, then $\gamma / \omega_{0}=1 / \sqrt{2}, I_{Q}=1 / 2$ and the harmonic term in equation (23) is zero.

The graphs of this motion are sketched in figure 9, where the anharmonicity is clearly visible in panel (b). In fact, the more the curve lies in the first and third quadrant, where $\ddot{\xi}$ has the same sign of $\xi$, the more the motion is anharmonic because the acting force has the opposite sign of a harmonic force. The body is accelerated towards the equilibrium position (A to B) in an initial brief interval of time and then it is slowed down.

If $\gamma / \omega_{0} \sim 1$ then:

$$
I_{Q} \sim 1 \text { and } T_{Q} \sim T / 2
$$

and the motion is practically just a coming back to the equilibrium position.

In conclusion, we stress the importance of analyzing the behavior of the force (acceleration) as a function of position to discriminate how much a damped motion is different from an ideal harmonic one. The physics of the problem is regulated by the ratio $\gamma / \omega_{0}$. If $\gamma / \omega_{0}$ is generally less then 0.16 (figure $7(\mathrm{~b})$ ), we can roughly say that the oscillation is substantially harmonic. On the contrary, when $\gamma / \omega_{0}$ is greater than $\frac{1}{\sqrt{2}}$ (figure 9(b)), a clear anharmonicity appears.

\section{Some didactical considerations}

From the previous analysis, it appears that it is often extremely simple to infer the anharmonicity/harmonicity of the small oscillations, even without knowing the exact dependence of the force on the displacement.

First of all, in the first approximation, linear damping does not substantially alter the harmonicity, provided some complete oscillations are clearly visible. Keeping in mind the four-point criterion of section 2, just looking at the motion and, if needed, also listening to the sound produced by the oscillations, allows us to label the oscillations as harmonic or not. If we have just a look at the motion of a bouncing disk, we immediately see that it does not have a stable equilibrium position; by simply watching the form of the track of a Galileo oscillator, we understand that in the equilibrium point there is a problem of continuity of force. Therefore, we immediately conclude that these motions are not harmonic. If one is not yet convinced, just by listening to the changing of the ticking rhythm, which reflects a dependence of the frequency on the amplitude, s(he) will finally be persuaded. Looking at the trajectory of the bob of an interrupted pendulum, an abrupt curvature change, as well as a sudden variation of the half-period, are evident. The case of the $x^{4}$-track is particularly intriguing, since viewing could not be enough, hearing could be necessary to realize the great dependence of the frequency on the amplitude.

From a laboratory point of view, a little warning is also worth mentioning; in fact, a restoring force of the form

$$
F_{\xi}(\xi)=-k_{1} \xi-k_{3} \xi^{3} ; \quad k_{1}>0 ; \quad k_{3}>0
$$

clearly satisfies the four-point criterion; nevertheless if $k_{3} \gg k_{1}$, anharmonic oscillations will appear as soon as the amplitude of oscillation is not small enough. This example shows that the observed anharmonicity/harmonicity of the small oscillations depends, in general, both on the experimental setup and on the measurement devices.

In a didactical path, it should be very instructive to ask students to try to understand at a glance the anharmonicity/harmonicity of two seesaws: a bar put on a cylindrical-shaped pivot 

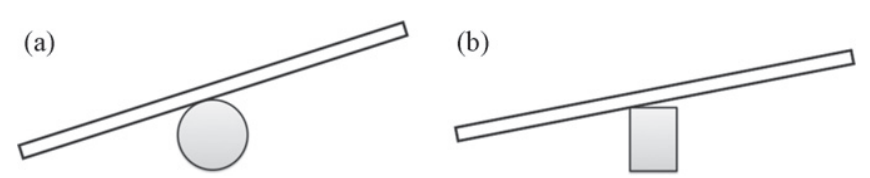

Figure 10. (a) The seesaw with a cylindrical-shaped pivot; (b) The seesaw with a square pivot.

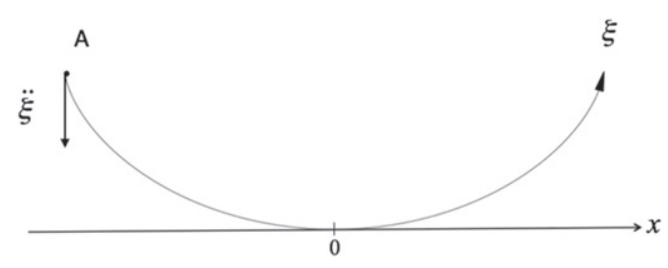

Figure 11. The cycloidal track.

(figure 10(a)) and a bar put on a square pivot (figure 10(b)) [13]. It should be clear that, for small oscillations, the former seesaw performs harmonic motion, while the latter does not (the eye sees an edge and the ear hears an increasingly frequent ticking).

As we have already said in subsection 3.3, while harmonicity implies isochronism, the reverse is not true. Let us consider a body moving on a cycloidal track under the effect of gravity (figure 11).

Neglecting friction, the motion is harmonic along the cycloid, whatever the amplitude. The equation of motion is [14]

$$
\ddot{\xi}+\frac{g}{4 R} \xi=0
$$

where $R$ is the radius of the circle generating the cycloid and $g$ the gravity acceleration. As a consequence, the oscillations are all isochronous. It is, therefore, straightforward to understand that even the $x$-component of the motion is isochronous for every amplitude. But it is harmonic only for small oscillations, while with increasing amplitude, the anharmonicity clearly appears. In fact, the invertible transformation from $\xi$ to $x$ is not linear and, therefore, it does not preserve the form of equation (29). To better understand this fact, we can consider an oscillation starting from point $\mathrm{A}$ in figure 11: the $x$-component of the acceleration is zero in that point, at variance to what a harmonic motion requires. For continuity reasons, the motion will therefore be anharmonic even in the intermediate amplitude region.

\section{Conclusions}

In order to gain a meaningful conceptual insight on harmonic motion, a careful definition joining mathematical rigor and physical intuition is needed. Starting from our experience with graduate students in mathematics, we made an educational reconstruction of some basic physics regarding oscillations that brought us to define the harmonic motion as the one that comes from the linearization around the equilibrium position of a suitable regular restoring force. This definition is particularly simple and useful in that it allows a quick identification of anharmonicity/harmonicity of the small oscillations even without explicitly knowing the 
equation of motion. For instance, there is no need to know the exact dependence of the force on the displacement to understand that the small oscillations of a pendulum are harmonic; nor it is necessary to know the exact force acting on a seesaw with a flat pivot to predict its anharmonicity, even for small oscillations.

Notwithstanding the simplicity and the great explicative power of the previous definition, some care must be taken in dealing with the relations among some mathematical subtleties and the physical reality. A four-point criterion, based on some mathematical considerations that are often disregarded, is very useful to discriminate between anharmonicity and harmonicity and allows a quick recognition of the motion features. Moreover, a careful analysis of damping is important in making students aware of the explicit interplay between theoretical thinking and practical measurements.

\section{Acknowledgements}

We are grateful to Tommaso Maccacaro for a careful reading of this paper and constructive comments.

\section{References}

[1] Fitzpatrick R 2013 Oscillations and Waves An Introduction (Boca Raton, FL: Taylor \& Francis)

[2] Mencuccini C and Silvestrini V 2013 Fisica 1. Meccanica termodinamica. Corso di fisica per le facoltà scientifiche. Con esempi ed esercizi (Napoli: Liguori Editore)

[3] Feynman R P, Leighton R B and Sands M The Feynman Lectures on Physics I (New York: Basic Books)

[4] Arnold V I 1979 Metodi Matematici della Meccanica Classica (Roma: Editori Riuniti)

[5] Barbieri S R and Giliberti M 2012 Laboratorio aperto oscillazioni e Onde (Milano: CUSL)

[6] Bergomi N et al 1997 Teaching mechanical oscillations using an integrated curriculum Int. J. Sci. Ed. 19 981-95

[7] Logger Pro 2013 Software and reference manual Retrieved from www.vernier.com/products/ software/lp/

[8] Tracker 2013 Software and reference manual Retrieved from www.cabrillo.edu/dbrown/tracker

[9] http://www.labtrek.it/torzo/oliphys/8/Galil8.htm

[10] Gil S and Di Gregorio D E 2003 Nonisochronism in the interrupted pendulum Am. J. Phys. 71 1115-20 and references therein

[11] Barratt C and Strobel G L 1981 Sliding friction and the harmonic oscillator Am. J. Phys. 49 500-1

[12] Onorato P, Mascoli D and DeAmbrosis A 2010 Damped oscillations and equilibrium in a massspring system subject to sliding friction forces: integrating experimental and theoretical analyses Am. J. Phys. 78 1120-7

[13] Pecori B and Torzo G 2001 Physics of the seesaw Phys. Teach. 39 25-29

[14] Onorato P, Mascheretti P and DeAmbrosis A 2013 Studying the motion along cycloidal paths by means of digital video analysis Eur. J. Phys. 34 921-30 